\title{
Belief that drug usage in the United States is increasing when it is really decreasing: An example of the availability heuristic
}

\author{
RUSSELL EISENMAN \\ McNeese State University, Lake Charles, Louisiana
}

\begin{abstract}
The availability heuristic is a cognitive rule of thumb in which probability of events is judged based on how readily they come to mind (Tversky \& Kahneman, 1974). This heuristic can lead to misjudgments when the event is emphasized in the media but actually occurs less frequently than would seem to be the case. The present study asked 104 college students if drug usage in the United States was increasing or decreasing over the past several years, and found they were significantly likely to say it was increasing $(p<.001)$. However, data from the National Household Survey on Drug Abuse, a nationwide sample of 9,259 subjects in households throughout the United States, shows that drug usage has been declining. The students' perceptions appear to show the working of the availability heuristic.
\end{abstract}

According to Tversky and Kahneman (1974), people often use shorthand methods for understanding the world. These heuristics include the availability heuristic, where probability of events is judged on how readily they come to mind, and the representativeness heuristic, where an event or object is seen as belonging to a category the more closely it resembles the typical instance. With regard to the availability heuristic, distorted perceptions could occur because an event is emphasized in the mass media. Tyler and Cook (1984) found evidence for this kind of distortion, due to media emphasis. A current media emphasis is on drugs. The United States has a "war on drugs," and the media often report about people arrested for drug possession or sales. Some newspapers carry daily stories of drug arrests, while there are several television shows known as "reality programming" in which reallife drug raids are shown. In one such reality program, American Detectives, almost every show is about drug raids, as opposed to, say, detectives investigating burglaries or murders.

\section{METHOD}

\section{Subjects}

The participants were 104 undergraduate students from two introduction to psychology classes at a state university. The data on actual drug usage was obtained from the National Household Survey on Drug Abuse, which involved 9,259 respondents in households in the contiguous United States.

\section{Procedure}

In the college classrooms, students were asked, "Is drug usage in the United States increasing or decreasing over the past several years?" They were urged to write down one of the two alternatives on a sheet of paper, but in response to a small number of questions, they were told that it was permissible to write "don't know" or "staying the same." Four subjects gave this kind of answer, and their data were not used.

Address requests for reprints to R. Eisenman, Department of Psychology, McNeese State University, Lake Charles, LA 70609-1895.
In past studies by the author, the vast majority of students do not choose the intermediate categories, except when it is something they feel ignorant about.

For the National Household Survey on Drug Abuse, begun in 1971 under the auspices of the National Commission on Marijuana and Drug Abuse and sponsored by the National Institute on Drug Abuse, 9,259 respondents were asked about their usage of both legal and illegal drugs. The survey sampled people 12 years of age and older living in households in the contiguous United States. Personal interviews and selfadministered answer sheets were used. To find out about drug usage, the following groups were purposely oversampled: blacks, Hispanics, young people, and residents of the metropolitan Washington, DC area. Otherwise, the sampling was representative of people living in households in the United States (with the exception of Alaska and Hawaii). Omitted from this survey of households was the homeless, people living in military installations (dormitories or other group quarters), and those in institutions such as hospitals or jails.

\section{Results}

For the college students, 70 said that drug usage is increasing and 30 reported that drug usage is decreasing $\left[\chi^{2}(1)=16, p<.001\right.$, two-tailed test $]$.

In comparison with the college students' belief that drug usage is increasing, the data from Tables 1-3 show that drug usage, for both legal and illegal drugs, has been decreasing in recent years. Table 1 reports the data in terms of lifetime prevalence of drug usage (i.e., whether the person has ever used the drug). Table 2 reports the data in terms of annual drug usage (i.e., usage within the past year). Table 3 reports the data in terms of usage within the past month. All three tables get at drug usage by the subject, but each covers a different time period. Thus, if a person used a drug 10 years ago but not since, it would show up in Table 1 (lifetime prevalence), but not in Tables 2 or 3 .

Although drug usage has been decreasing in recent years, according to the survey data, there are still many millions of Americans who use drugs, as shown in Table 4. Thus, "decreasing" does not mean "nonexistent." 


\section{DISCUSSION}

The students would seem to have been the victims of the availability heuristic. Since there is so much in the mass media about drug usage, the students judge that drug usage must be increasing. Mass media can at times, result in people's misperception (Tyler \& Cook, 1984), as well as, at other times, people's correct perception of things. Since acts may be judged negatively due to the perception of their social importance (Eisenman, 1991), the misjudgments due to heuristics can influence whether or not a person or a group is judged as acceptable or unacceptable. Note that these judgments may be based on faulty reasoning (e.g., if a marijuana smoker is judged as being as dangerous to society as a cocaine user), due to the media's reporting sensationalistic stories about each, or if a person who belongs to a minority political party is judged as being like a Communist, because the person doing the judging has faulty reasoning about these concepts. Thus, heuristics can lead to judgments of deviance and the ensuing hatred (Eisenman, 1991).

This article assumes that the government data are correct and that the student judgments are incorrect. Is it possible it could be the other way around? While it seems unlikely, it should be considered that the students may have access to data the government surveys do not reveal. For ex- ample, the students may know, from personal experience, that use of drugs is increasing, while respondents to the government survey may give dishonest answers. In this scenario, the students would be correct, and the results would be explained without using the heuristics concept.

It would be possible to ask the students about drug usage in more detailed fashion. For example, they could be asked about specific drugs, with a questionnaire that mentions each drug and has the student rate its usage as "increasing" or "decreasing." Or, the students could be asked to rate the increasing-decreasing information on a 1-7 scale, so that intermediate answers would not be discouraged. Also, it might be useful to counterbalance the order of the question used in the present study, so that some subjects are first asked about a decrease in drug usage and then asked about an increase in drug usage, to control for possible order effects.

The study seems to show the operation of the availability heuristic (Tversky \& Kahneman, 1974). Students think drug usage has been increasing, while nationwide surveys suggest that in recent years drug usage is decreasing. The mass media would seem to be the likely basis of this student misperception, but educational classes on drug abuse and the frequent comments by people in authority might also contribute. Also, as Table 4 shows, drug usage is widespread in the United States, and this may influence the students' perceptions. It might be difficult to think that something so widespread is, at the same time, decreasing in frequency.

Table 1

Lifetime Prevalence (in Percent) of Drug use: 1972 to 1990

\begin{tabular}{|c|c|c|c|c|c|c|c|c|c|}
\hline & 1972 & 1974 & 1976 & 1977 & 1979 & 1982 & 1985 & 1988 & 1990 \\
\hline \multicolumn{10}{|c|}{ Youth (Age 12-17) } \\
\hline Marijuana & 14.0 & 23.0 & 22.4 & 28.0 & 30.9 & 26.7 & 23.6 & 17.4 & 14.8 \\
\hline Hallucinogens & 4.8 & 6.0 & 5.1 & 4.6 & 7.1 & 5.2 & 3.3 & 3.5 & 3.3 \\
\hline Cocaine & 1.5 & 3.6 & 3.4 & 4.0 & 5.4 & 6.5 & 4.9 & 3.4 & 2.6 \\
\hline Heroin & 0.6 & 1.0 & 0.5 & 1.1 & 0.5 & $*$ & $*$ & 0.6 & 0.7 \\
\hline \multicolumn{10}{|c|}{ Nonmedical Use of: } \\
\hline Stimulants & 4.0 & 5.0 & 4.4 & 5.2 & 3.4 & 6.7 & 5.6 & 4.2 & 4.5 \\
\hline Sedatives & 3.0 & 5.0 & 2.8 & 3.1 & 3.2 & 5.8 & 4.1 & 2.4 & 3.3 \\
\hline Tranquilizers & 3.0 & 3.0 & 3.3 & 3.8 & 4.1 & 4.9 & 4.8 & 2.0 & 2.7 \\
\hline Analgesics & - & - & - & - & 3.2 & 4.2 & 5.8 & 4.2 & 6.5 \\
\hline Alcohol & - & 54.0 & 53.6 & 52.6 & 70.3 & 65.2 & 55.5 & 50.2 & 48.2 \\
\hline Cigarettes & - & 52.0 & 45.5 & 47.3 & 54.1 & 49.5 & 45.2 & 42.3 & 40.2 \\
\hline Any Illicit Use & - & - & - & - & 34.3 & 27.6 & 29.5 & 24.7 & 22.7 \\
\hline \multicolumn{10}{|c|}{ Young Adults (Age 18-25) } \\
\hline Marijuana & 47.9 & 52.7 & 52.9 & 59.9 & 68.2 & 64.1 & 60.3 & 56.4 & 52.2 \\
\hline Hallucinogens & - & 16.6 & 17.3 & 19.8 & 25.1 & 21.1 & 11.3 & 13.8 & 12.0 \\
\hline Cocaine & 9.1 & 12.7 & 13.4 & 19.1 & 27.5 & 28.3 & 25.2 & 19.7 & 19.4 \\
\hline Heroin & 4.6 & 4.5 & 3.9 & 3.6 & 3.5 & 1.2 & 1.2 & 0.4 & 0.6 \\
\hline \multicolumn{10}{|c|}{ Nonmedical Use of: } \\
\hline Stimulants & 12.0 & 17.0 & 16.6 & 21.2 & 18.2 & 18.0 & 17.1 & 11.3 & 9.0 \\
\hline Sedatives & 10.0 & 15.0 & 11.9 & 18.4 & 17.0 & 18.7 & 11.0 & 5.5 & 4.0 \\
\hline Tranquilizers & 7.0 & 10.0 & 9.1 & 13.4 & 15.8 & 15.1 & 12.0 & 7.8 & 5.9 \\
\hline Analgesics & - & - & - & - & 11.8 & 12.1 & 11.3 & 9.4 & 8.1 \\
\hline Alcohol & - & 81.6 & 83.6 & 84.2 & 95.3 & 94.6 & 92.6 & 90.3 & 88.2 \\
\hline Cigarettes & - & 68.8 & 70.1 & 67.6 & 82.8 & 76.9 & 75.6 & 75.0 & 70.5 \\
\hline Any Illicit Use & - & - & - & - & 69.9 & 65.3 & 64.3 & 58.9 & 55.8 \\
\hline \multicolumn{10}{|c|}{ Older Adults (Age 26+) } \\
\hline Marijuana & 7.4 & 9.9 & 12.9 & 15.3 & 19.6 & 23.0 & 27.2 & 30.7 & 31.8 \\
\hline Hallucinogens & - & 1.3 & 1.6 & 2.6 & 4.5 & 6.4 & 6.2 & 6.6 & 7.4 \\
\hline Cocaine & 1.6 & 0.9 & 1.6 & 2.6 & 4.3 & 8.5 & 9.5 & 9.9 & 10.9 \\
\hline Heroin & $*$ & 0.5 & 0.5 & 0.8 & 1.0 & 1.1 & 1.1 & 1.1 & 0.9 \\
\hline \multicolumn{10}{|c|}{ Nonmedical Use of: } \\
\hline Stimulants & 3.0 & 3.0 & 5.6 & 4.7 & 5.8 & 6.2 & 7.9 & 6.6 & 6.9 \\
\hline Sedatives & 2.0 & 2.0 & 2.4 & 2.8 & 3.5 & 4.8 & 5.2 & 3.3 & 3.7 \\
\hline Tranquilizers & 5.0 & 2.0 & 2.7 & 2.6 & 3.1 & 3.6 & 7.2 & 4.6 & 4.2 \\
\hline Analgesics & - & - & - & - & 2.7 & 3.2 & 5.6 & 4.5 & 5.1 \\
\hline Alcohol & - & 73.2 & 74.7 & 77.9 & 91.5 & 88.2 & 89.4 & 88.6 & 86.8 \\
\hline Cigarettes & - & 65.4 & 64.5 & 67.0 & 83.0 & 78.7 & 80.5 & 79.6 & 78.0 \\
\hline Any Illicit Use & - & - & - & - & 23.0 & 24.7 & 31.5 & 33.7 & 35.3 \\
\hline
\end{tabular}

Note-Data taken from the 1990 National Household Survey on Drug Abuse. - No estimate available. *Low precision; no estimate shown. 
Table 2

Annual Drug Use (in Percent): 1972 to 1990

\begin{tabular}{|c|c|c|c|c|c|c|c|c|c|}
\hline & 1972 & 1974 & 1976 & 1977 & 1979 & 1982 & 1985 & 1988 & 1990 \\
\hline \multicolumn{10}{|c|}{ Youth (Age 12-17) } \\
\hline Marijuana & - & 18.5 & 18.4 & 22.3 & 24.1 & 20.6 & 19.7 & 12.6 & 11.3 \\
\hline Hallucinogens & 3.6 & 4.3 & 2.8 & 3.1 & 4.7 & 3.6 & 2.7 & 2.8 & 2.4 \\
\hline Cocaine & 1.5 & 2.7 & 2.3 & 2.6 & 4.2 & 4.1 & 4.0 & 2.9 & 2.2 \\
\hline Heroin & $*$ & $*$ & $*$ & 0.6 & $*$ & $*$ & $*$ & 0.4 & 0.6 \\
\hline \multicolumn{10}{|c|}{ Nonmedical Use of: } \\
\hline Stimulants & - & 3.0 & 2.2 & 3.7 & 2.9 & 5.6 & 4.3 & 2.8 & 3.0 \\
\hline Sedatives & - & 2.0 & 1.2 & 2.0 & 2.2 & 3.7 & 2.9 & 1.7 & 2.2 \\
\hline Tranquilizers & - & 2.0 & 1.8 & 2.9 & 2.7 & 3.3 & 3.4 & 1.6 & 1.5 \\
\hline Analgesics & - & - & - & - & 2.2 & 3.7 & 3.8 & 3.0 & 4.8 \\
\hline Alcohol & - & 51.0 & 49.3 & 47.5 & 53.6 & 52.4 & 51.7 & 44.6 & 41.0 \\
\hline Cigarettes & - & - & - & - & $13.3 \dagger$ & 24.8 & 25.8 & 22.8 & 22.2 \\
\hline Any Illicit Use & - & - & - & - & 26.0 & 22.0 & 23.7 & 16.8 & 15.9 \\
\hline \multicolumn{10}{|c|}{ Young Adults (Age 18-25) } \\
\hline Marijuana & - & 34.2 & 35.0 & 38.7 & 46.9 & 40.4 & 36.9 & 27.9 & 24.6 \\
\hline Hallucinogens & - & 6.1 & 6.0 & 6.4 & 9.9 & 6.9 & 4.0 & 5.6 & 3.9 \\
\hline Cocaine & - & 8.1 & 7.0 & 10.2 & 19.6 & 18.8 & 16.3 & 12.1 & 7.5 \\
\hline Heroin & - & 0.8 & 0.6 & 1.2 & 0.8 & $*$ & 0.6 & 0.3 & 0.5 \\
\hline \multicolumn{10}{|c|}{ Nonmedical Use of: } \\
\hline Stimulants & - & 8.0 & 8.8 & 10.4 & 10.1 & 10.8 & 9.9 & 6.4 & 3.4 \\
\hline Sedatives & - & 4.2 & 5.7 & 8.2 & 7.3 & 8.7 & 5.0 & 3.3 & 2.0 \\
\hline Tranquilizers & - & 4.6 & 6.2 & 7.8 & 7.1 & 5.9 & 6.4 & 4.6 & 2.4 \\
\hline Analgesics & - & - & - & - & 5.2 & 4.4 & 6.6 & 5.5 & 4.1 \\
\hline Alcohol & - & 77.1 & 77.9 & 79.8 & 86.6 & 87.1 & 87.2 & 81.7 & 80.2 \\
\hline Cigarettes & - & - & - & - & $46.7 \dagger$ & 47.2 & 44.3 & 44.7 & 39.7 \\
\hline Any Illicit Use & - & - & - & - & 49.4 & 43.4 & 42.6 & 32.0 & 28.7 \\
\hline \multicolumn{10}{|c|}{ Older Adults (Age 26+) } \\
\hline Marijuana & - & 3.8 & 5.4 & 6.4 & 9.0 & 10.6 & 9.5 & 6.9 & 7.3 \\
\hline Hallucinogens & - & $*$ & $*$ & $*$ & 0.5 & 0.8 & 1.0 & 0.6 & 0.4 \\
\hline Cocaine & - & $*$ & 0.6 & 0.9 & 2.0 & 3.8 & 4.2 & 2.7 & 2.4 \\
\hline Heroin & - & $*$ & $*$ & $*$ & $*$ & $*$ & $*$ & 0.3 & 0.1 \\
\hline \multicolumn{10}{|c|}{ Nonmedical Use of: } \\
\hline Stimulants & - & * & 0.8 & 0.8 & 1.3 & 1.7 & 2.6 & 1.7 & 1.0 \\
\hline Sedatives & - & * & 0.6 & $*$ & 0.8 & 1.4 & 2.0 & 1.2 & 0.8 \\
\hline Tranquilizers & - & $*$ & 1.2 & 1.1 & 0.9 & 1.1 & 2.8 & 1.8 & 1.0 \\
\hline Analgesics & - & - & - & - & 0.5 & 1.0 & 2.9 & 2.1 & 1.9 \\
\hline Alcohol & - & 62.7 & 64.2 & 65.8 & 72.4 & 72.0 & 73.6 & 68.6 & 66.6 \\
\hline Cigarettes & - & - & - & - & $39.7 \dagger$ & 38.2 & 36.0 & 33.7 & 31.9 \\
\hline Any Illicit Use & - & - & - & - & 10.0 & 11.8 & 13.3 & 10.2 & 10.0 \\
\hline
\end{tabular}

Note-Data taken from the 1990 National Household Survey on Drug Abuse. - No estimate available. *Low precision; no estimate shown. †Includes only people who ever smoked at least five packs.

Table 3

Current (Past Month) Drug Use (in Percent): 1972 to 1990

\begin{tabular}{|c|c|c|c|c|c|c|c|c|c|}
\hline & 1972 & 1974 & 1976 & 1977 & 1979 & 1982 & 1985 & 1988 & 1990 \\
\hline \multicolumn{10}{|c|}{ Youth (Age 12-17) } \\
\hline Marijuana & 7.0 & 12.0 & 12.3 & 16.6 & 16.7 & 11.5 & 12.0 & 6.4 & 5.2 \\
\hline Hallucinogens & 1.4 & 1.3 & 0.9 & 1.6 & 2.2 & 1.4 & 1.2 & 0.8 & 0.9 \\
\hline Cocaine & 0.6 & 1.0 & 1.0 & 0.8 & 1.4 & 1.6 & 1.5 & 1.1 & 0.6 \\
\hline Heroin & $*$ & $*$ & $*$ & $*$ & $*$ & $*$ & $*$ & $*$ & $*$ \\
\hline \multicolumn{10}{|c|}{ Nonmedical Use of: } \\
\hline Stimulants & - & 1.0 & 1.2 & 1.3 & 1.2 & 2.6 & 1.6 & 1.2 & 1.0 \\
\hline Sedatives & - & 1.0 & $*$ & 0.8 & 1.1 & 1.3 & 1.0 & 0.6 & 0.9 \\
\hline Tranquilizers & - & 1.0 & 1.1 & 0.7 & 0.6 & 0.9 & 0.6 & 0.2 & 0.5 \\
\hline Analgesics & - & - & - & - & 0.6 & 0.7 & 1.6 & 0.9 & 1.4 \\
\hline Alcohol & - & 34.0 & 32.4 & 31.2 & 37.2 & 30.2 & 31.0 & 25.2 & 24.5 \\
\hline Cigarettes & - & 25.0 & 23.4 & 22.3 & $12.1 \dagger$ & 14.7 & 15.3 & 11.8 & 11.6 \\
\hline Any Illicit Use & - & - & - & - & 17.6 & 12.7 & 14.9 & 9.2 & 8.1 \\
\hline \multicolumn{10}{|c|}{ Young Adults (Age 18-25) } \\
\hline Marijuana & 27.8 & 25.2 & 25.0 & 27.4 & 35.4 & 27.4 & 21.8 & 15.5 & 12.7 \\
\hline Hallucinogens & - & 2.5 & 1.1 & 2.0 & 4.4 & 1.7 & 1.9 & 1.9 & 0.8 \\
\hline Cocaine & - & 3.1 & 2.0 & 3.7 & 9.3 & 6.8 & 7.6 & 4.5 & 2.2 \\
\hline Heroin & - & $*$ & $*$ & $*$ & $*$ & $*$ & $*$ & $*$ & $*$ \\
\hline
\end{tabular}


Table 3 (Continued)

\begin{tabular}{|c|c|c|c|c|c|c|c|c|c|}
\hline & 1972 & 1974 & 1976 & 1977 & 1979 & 1982 & 1985 & 1988 & 1990 \\
\hline \multicolumn{10}{|c|}{ Nonmedical Use of: } \\
\hline Stimulants & - & 3.7 & 4.7 & 2.5 & 3.5 & 4.7 & 3.7 & 2.4 & 1.2 \\
\hline Sedatives & - & 1.6 & 2.3 & 2.8 & 2.8 & 2.6 & 1.6 & 0.9 & 0.7 \\
\hline Tranquilizers & - & 1.2 & 2.6 & 2.4 & 2.1 & 1.6 & 1.6 & 1.0 & 0.5 \\
\hline Analgesics & - & - & - & - & 1.0 & 1.0 & 1.8 & 1.5 & 1.2 \\
\hline Alcohol & - & 69.3 & 69.0 & 70.0 & 75.9 & 70.9 & 71.4 & 65.3 & 63.3 \\
\hline Cigarettes & - & 48.4 & 49.4 & 47.3 & $42.6 \dagger$ & 39.5 & 36.8 & 35.2 & 31.5 \\
\hline Any Illicit Use & - & - & - & - & 37.1 & 30.4 & 25.7 & 17.8 & 14.9 \\
\hline \multicolumn{10}{|c|}{ Older Adults (Age 26+) } \\
\hline Marijuana & 2.5 & 2.0 & 3.5 & 3.3 & 6.0 & 6.6 & 6.1 & 3.9 & 3.6 \\
\hline Hallucinogens & - & $*$ & $*$ & $*$ & $*$ & $*$ & $*$ & $*$ & 0.1 \\
\hline Cocaine & - & * & * & * & 0.9 & 1.2 & 2.0 & 0.9 & 0.6 \\
\hline Heroin & - & * & * & * & $*$ & $*$ & $*$ & $*$ & $*$ \\
\hline \multicolumn{10}{|c|}{ Nonmedical Use of: } \\
\hline Stimulants & - & * & * & 0.6 & 0.5 & 0.6 & 0.7 & 0.5 & 0.3 \\
\hline Sedatives & - & * & 0.5 & * & * & $*$ & 0.6 & 0.3 & 0.1 \\
\hline Tranquilizers & - & $*$ & $*$ & $*$ & $*$ & $*$ & 1.0 & 0.6 & 0.2 \\
\hline Analgesics & - & - & - & - & * & * & 0.9 & 0.4 & 0.6 \\
\hline Alcohol & - & 54.5 & 56.0 & 54.9 & 61.3 & 59.8 & 60.6 & 54.8 & 52.3 \\
\hline Cigarettes & - & 39.1 & 38.4 & 38.7 & $36.9 \dagger$ & 34.6 & 32.8 & 29.8 & 27.7 \\
\hline Any Illicit Use & - & - & - & - & 6.5 & 7.5 & 8.5 & 4.9 & 4.6 \\
\hline
\end{tabular}

Note-Data taken from the 1990 National Household Survey on Drug Abuse. - No estimate available. *Low precision; no estimate shown. †Includes only people who ever smoked at least five packs.

Table 4

Population Estimates of Lifetime and Current Drug Use, 1990

\begin{tabular}{|c|c|c|c|c|c|c|c|c|c|c|c|c|c|c|c|c|}
\hline & \multicolumn{4}{|c|}{$\begin{array}{c}12-17 \text { years } \\
\text { (pop. } 19,977,918 \text { ) }\end{array}$} & \multicolumn{4}{|c|}{$\begin{array}{c}18-25 \text { years } \\
\text { (pop. } 29,020,582 \text { ) }\end{array}$} & \multicolumn{4}{|c|}{$\begin{array}{c}26+\text { years } \\
\text { (pop. } 152,189,483 \text { ) }\end{array}$} & \multicolumn{4}{|c|}{$\begin{array}{c}\text { Total } \\
\text { (pop. 201,187,983) }\end{array}$} \\
\hline & $\%$ & $\begin{array}{c}\text { Ever } \\
\text { Used } \\
n\end{array}$ & $\%$ & $\begin{array}{c}\text { Current } \\
\text { User } \\
n\end{array}$ & $\%$ & $\begin{array}{c}\text { Ever } \\
\text { Used } \\
n\end{array}$ & $\%$ & $\begin{array}{c}\text { Current } \\
\text { User } \\
n\end{array}$ & $\%$ & $\begin{array}{c}\text { Ever } \\
\text { Used } \\
n\end{array}$ & $\%$ & $\begin{array}{c}\text { Current } \\
\text { User } \\
n\end{array}$ & $\%$ & $\begin{array}{c}\text { Ever } \\
\text { Used } \\
n\end{array}$ & $\%$ & $\begin{array}{c}\text { Current } \\
\text { User } \\
n\end{array}$ \\
\hline $\begin{array}{l}\text { Marijuana } \\
\text { and Hashish }\end{array}$ & 15 & $2,954,000$ & 51 & $1,030,000$ & 52 & $15,140,0001$ & 13 & $3,692,000$ & 32 & $48,413,000$ & 4 & $5,483,000$ & 33 & $66,507,000$ & 6 & $10,206,000$ \\
\hline Hallucinogens & 3 & 652,000 & 1 & 186,000 & 12 & $3,485,000$ & 1 & 243,000 & 7 & $11,203,000$ & $*$ & * & 8 & $15,339,000$ & $*$ & 553,000 \\
\hline Inhalants & 8 & $1,548,000$ & 2 & 441,000 & 10 & $3,019,000$ & 1 & 344,000 & 4 & $5,729,000$ & $*$ & * & 5 & $10,296,000$ & 1 & $1,188,000$ \\
\hline Cocaine & 3 & 518,000 & 1 & 115,000 & 19 & $5,620,000$ & 2 & 630,000 & 11 & $16,601,000$ & 1 & 856,000 & 11 & $22,739,000$ & 1 & $1,601,000$ \\
\hline Crack & 1 & 201,000 & $*$ & 45,000 & 3 & 802,000 & 1 & 192,000 & 1 & $1,755,000$ & $*$ & $*$ & 1 & $2,757,000$ & $*$ & 494,000 \\
\hline Heroin & 1 & 145,000 & $*$ & 7,000 & 1 & 166,000 & $*$ & 25,000 & 1 & $1,343,000$ & $*$ & $*$ & 1 & $1,654,000$ & $*$ & 48,000 \\
\hline Stimulants & 5 & 898,000 & 1 & 191,000 & 9 & $2,621,000$ & 1 & 350,000 & 7 & $10,444,000$ & $*$ & $*$ & 7 & $13,963,000$ & 1 & 957,000 \\
\hline Sedatives & 3 & 658,000 & 1 & 182,000 & 4 & $1,157,000$ & 1 & 215,000 & 4 & $5,700,000$ & $*$ & $*$ & 4 & $7,515,000$ & $*$ & 568,000 \\
\hline Tranquilizers & 3 & 533,000 & 1 & 110,000 & 6 & $1,702,000$ & 1 & 151,000 & 4 & $6,433,000$ & $*$ & $*$ & 4 & $8,668,000$ & $*$ & 568,000 \\
\hline Analgesics & 7 & $1,292,000$ & 1 & 274,000 & 8 & $2,349,000$ & 1 & 340,000 & 5 & $7,766,000$ & 1 & 923,000 & 6 & $11,408,000$ & 1 & $1,536,000$ \\
\hline Alcohol & 48 & $9,636,000$ & 254 & $4,895,000$ & 882 & $25,599,0006$ & 631 & $18,368,000$ & 871 & $132,145,0005$ & 527 & $79,656,000$ & 83 & $167,380,0005$ & 511 & $102,919,000$ \\
\hline & 40 & $8,041,000$ & 122 & $2,327,000$ & 712 & $20,468,0003$ & 32 & $9,143,000$ & 781 & $118,733,0002$ & 284 & $42,162,000$ & 73 & $147,241,0002$ & 27 & $53,633,000$ \\
\hline Smokeless & 12 & $2,356,000$ & 4 & 775,000 & 22 & $6,306,000$ & 6 & $1,734,000$ & 13 & $19,710,000$ & 3 & $4,602,000$ & 14 & $28,372,000$ & 4 & $7,111,000$ \\
\hline
\end{tabular}

Note-Data taken from the 1990 National Household Survey on Drug Abuse. Ever Used=used at least once in a person's lifetime. Current User $=$ used at least once in the 30 days prior to survey. *Amounts of less than $5 \%$ are not listed.

\section{REFERENCES}

Eisenman, R. (1991). From crime to creativity: Psychological and social factors in deviance. Dubuque, IA: Kendall/Hunt.

TVERSKY, A., \& KAHNEMAN, D. (1974). Judgment under uncertainty: Heuristics and biases. Science, 185, 1124-1131.
Tyler, T. R., \& Cook, F. L. (1984). The mass media and judgments of risk: Distinguishing impact on personal and societal level judgments. Journal of Personality and Social Psychology, 47, 693-708.

(Manuscript received January 6, 1993.) 